\title{
Growth Gharacteristics of Some Gram-negative Bacteria
}

\author{
By F. P. ERRINGTON, E. O. POWELL AND N. THOMPSON \\ Microbiological Research Establishment, Porton, \\ $\mathrm{Nr}$ Salisbury, Wiltshire
}

(Received 4 November 1964)

\begin{abstract}
SUMMARY
The growth of individual organisms of four Gram-negative species has been measured. The growth rate of the individual was not in general constant, and its mean value differed from one organism to another in the same culture. There was positive correlation between the size of an organism at termination and the size of its mother at termination. These findings are contrary to the major postulates of the Koch \& Schaechter hypothesis, but are so far no more than qualitatively established. Fission is unsymmetrical in respect of growth rate and size; the growth rate and size of sisters differ systematically according to their relative positions in the family tree.
\end{abstract}

\section{INTRODUCTION}

In this paper we record some measurements of the growth of individual organisms, indicate a number of seeming regularities in the pattern of growth, and compare our findings with the postulates of the Koch \& Schaechter (1962) hypothesis. Koch \& Schaechter compared some of the derivative consequences of the hypothesis with experimental results obtained by Schaechter, Williamson, Hood \& Koch (1962) but neither group of authors seems to have made a direct test of the postulates themselves. In pointing out deficiencies in the hypothesis we must not be thought captious; it is not to be expected at present that any 'model' of bacterial growth will agree more than very roughly with the known phenomena. In our opinion the Koch \& Schaechter hypothesis is likely to prove at least as fruitful and suggestive as any older view, and it certainly deserves as much consideration.

We have worked entirely with rod-like organisms, and in common with many authors we have accepted the approximation of taking the length of an organism as a measure of its volume. Collins \& Richmond (1962) discussed the difficulty of measuring volume accurately, and Powell \& Errington (1963b) showed that there can be detectable differences between the diameters of organisms drawn from the same steadily growing culture. Our curves for the lengthwise growth are thus not strictly paralleled by the true curves of growth in volume.

\section{EXPERIMENTAL METHOD}

Our numerical results consist of repeated measurements of length made at known times on single organisms in steadily growing cultures. The measurements were made with a Dyson image-splitting eyepiece (Dyson, 1959, 1960) in exactly the same way and under the same conditions as those of Powell \& Errington (1963b). Six series of experiments were carried out (Table 1) with four species and two media: tryptic 
Table 1. Organisms and growth conditions

Series
reference
no.
Sm 1
Pm 1
Ec 1
Ec. 2
Pa 1
Pa 2

$\quad$ Organism
Serratia marcescens
Proteus morganii
Escherichia coli
E. coli
Pseudomonas aeruginosa
P. aeruginosa

$\begin{array}{cc}\text { Medium } & \text { Temperature } \\ \text { TMB } & \mathbf{3 5}^{\circ} \\ \text { TMB } & \mathbf{3 0}^{\circ} \\ \text { TMB } & \mathbf{3 0}^{\circ} \\ \text { TMB } & \mathbf{3 0}^{\circ} \\ \text { TMB } & \mathbf{3 5}^{\circ} \\ \text { H 12P } & \mathbf{3 0}^{\circ}\end{array}$

meat broth (TMB) and a chemically defined medium (H 12P) described by Powell \& Errington (1963a). The Escherichia coli in series Ec 1 had at the time been recently isolated from human faeces. In series Ec 2, done a year later with nominally the same strain, the organisms grew more slowly and were larger and less uniform in size. It was discovered independently that the antigenic structure of the strain altered greatly on serial subculture; we therefore feel justified in treating the two series separately. The long generation times (mean 52 min.) in series $\mathrm{Pa} 2$ enabled us to make 50-100 length measurements during the life-span of an organism; no special details of the law of growth were thereby brought to light. Young cultures of Proteus morganii contained many filamentous organisms which broke down or were diluted out as the culture aged. Few remained in the inocula transferred to the culture chamber; organisms of moderate size were selected for examination, and their progeny, as far as we traced them, behaved regularly (Table 2).

The measurements in a typical experiment were conducted as indicated in Fig. 1; they were begun on a conveniently situated organism which was followed to its termination, then continued on one of its daughters, and so on, until crowding made the measurements difficult and inaccurate. The process was repeated on other

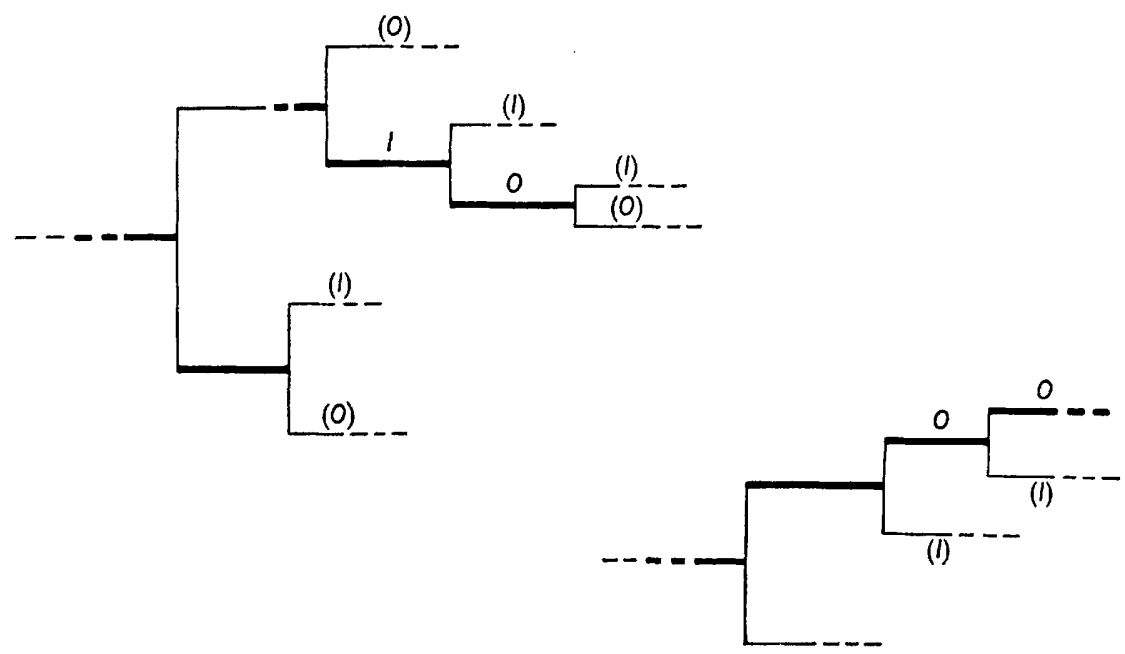

Fig. 1. Scheme of observations $n$ a typical experiment. Measurements were made on the organisms represented by heavy horizontal lines. The organisms are marked $I$ (inner) or $O$ (outer) according to their relative position in the family tree. 
parent organisms. Each experiment provided data on 1-3 such sequences of organisms. Powell \& Errington (1963a) showed that rod-like organisms might be classified as 'inner' $(I)$ or 'outer' $(O)$ according to their position in the family tree. We therefore recorded, where it was known, the position symbol $(I$ or $O)$ of each of the organisms measured.

A few experiments were also made to provide additional information on the relative sizes of sisters at inception. The measurements, made alternately on the members of each pair for a short time only, gave estimates of initial size probably less accurate than the rest, and these are not included in column 3 of Table 2.

\section{RESULTS}

\section{Growth of individual organisms}

There are two systematic errors of which we shall have to take account in treating length measurements as equivalent to volume measurements. First, some erratic changes in length occur at fission, and these can be ascribed in part to the rounding of the ends of the newly-formed organisms, the sum of whose lengths is always (in our experience) greater than the length of the mother (see also Powell \& Errington, $1963 b)$. Second, the length $(x)$ is not directly proportional to the volume $(v)$. The shape of the organism is roughly that of a cylinder terminated by hemispherical caps, and

$$
v=\pi r^{2}(x-2 r / 3)
$$

where $r$ is the transverse radius. If the increase in volume is strictly exponential with growth rate $\nu$,

and we find

$$
\frac{d \log v}{d t}=\nu
$$

$$
\frac{d \log x}{d t}=\nu(1-2 r / 3 x)
$$

That is, the rate of increase of $\log x$ increases with $x$. At worst, when the length $\left(x_{i}\right)$ at inception is $2 r$ and the organism is then a sphere, the acceleration is very small (Fig. 2); it is probably of no account in our work, in which no $x_{i}$ was less than $4 r$. We noted, however, that positive values of $d^{2} \log x / d t^{2}$ were more frequent than negative values.

We found that in most instances the logarithm of the length of an organism was a nearly linear function of time $(t)$, but in some the plot was certainly curved-the curvature could take either sense (Fig. 3). Viable organisms having a strongly curved characteristic were not obviously remarkable in other ways, and there was therefore no point in establishing limits of statistical significance for the various degrees of curvature.

With some exceptions we fitted an equation of the form

$$
\log x=\alpha+\beta t+\gamma t^{2}
$$

to the set of measurements on each organism. The fitting was carried out by the method of weighted least squares, based on the assumption that the random error in measuring $x$ was independent of the absolute value of $x$. The coefficients $\alpha, \beta, \gamma$ were thus determined by the three normal equations

$$
\Sigma t_{r}^{n} x_{r}^{2} \log x_{r}=\alpha \Sigma t_{r}^{n} x_{r}^{2}+\beta \Sigma t_{r}^{n+1} x_{r}^{2}+\gamma \Sigma t_{r}^{n+2} x_{r}^{2} \quad(n=0,1,2)
$$


in which the $x_{r}$ are the measured lengths at corresponding times $t_{r}$. The normal equations were solved for each organism by means of an electronic digital computer. The computer was so programmed as to yield, besides the three coefficients $\alpha, \beta, \gamma$ the residual

$$
x_{r}-\exp \left(\alpha+\beta t_{r}+\gamma t_{r}^{2}\right)
$$

at each measured value, the root-mean-square (r.m.s.) residual, and the estimated length of the organism at inception $\left(x_{i}\right)$ and termination $\left(x_{t}^{\prime}\right)$, the times $t_{i}, t_{t}$ of these events being known:

$$
x_{i}, x_{i}^{\prime}=\exp \left(\alpha+\beta t_{i, t}+\gamma t_{i, t}^{2}\right)
$$

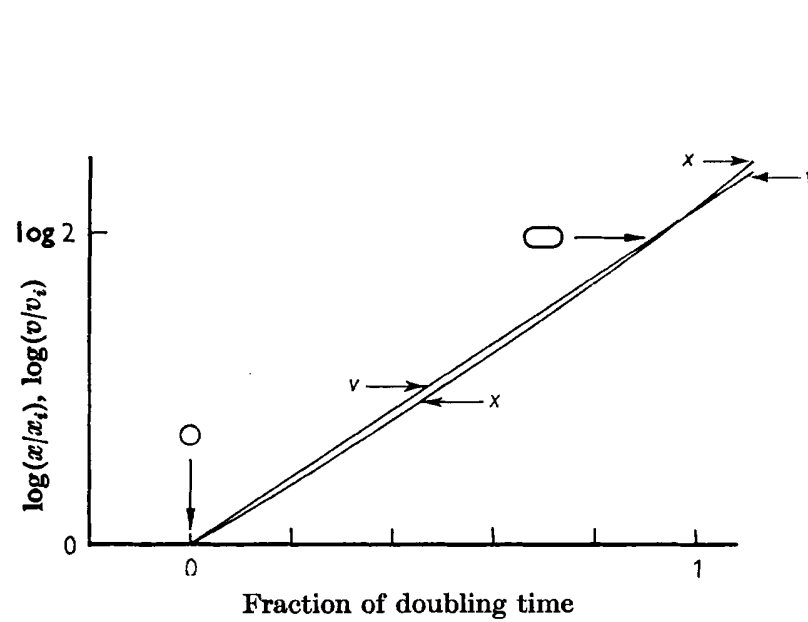

Fig. 2

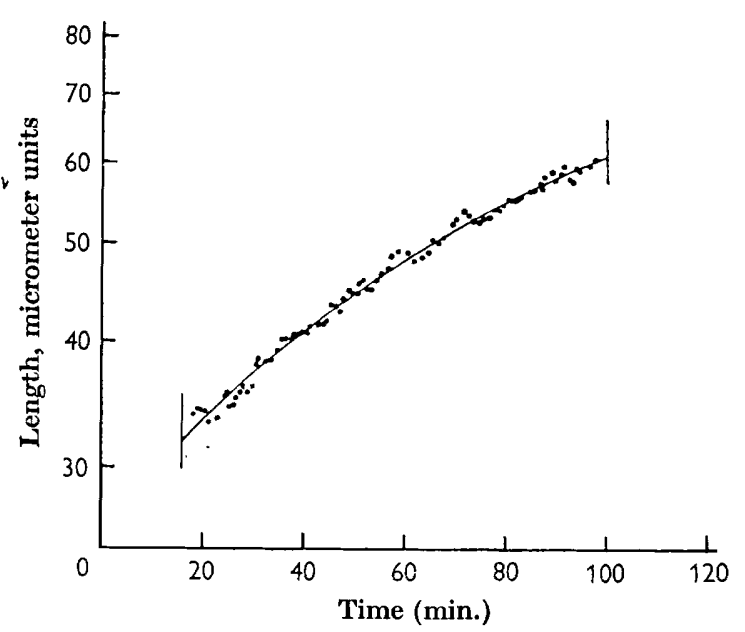

Fig. 3

Fig. 2. Growth of an organism initially spherical. If the law of growth in volume (v) is strictly exponential, the rate of growth in length $(x)$ is slightly accelerated.

Fig. 3. An organism of Pseudomonas aeruginosa showing a diminishing growth rate (series $\mathrm{Pa} 2$ ). The vertical bars indicate the times of inception and termination, and the continuous line was obtained by fitting equation (1) to the observations. The deviation from linearity, $\Delta$, is $-0 \cdot 113$. Logarithmic vertical scale.

Our results included some sequences of measurements covering only part of the life-span of an organism: its approach to termination or its growth for a short time after inception. Such sequences were used only to obtain estimates of $x_{i}$ or $x_{t}$; those which included less than sixteen $t_{r}, x_{r}$ pairs of co-ordinates were fitted to a simpler equation

$$
\log x=\alpha+\beta t
$$

in order not to embarrass a $\chi^{2}$ test, subsequently applied, for the overall goodness of fit of equation (1).

The grand r.m.s. residual from equation (1) for the total of 6000 length measurements was $0.0762 \mu$, i.e. about $2 \frac{1}{2}$ times the standard deviation to be expected from the Dyson eyepiece under the best conditions (Powell \& Errington, 1963b). The circumstances of the experiments were such that the full sensitivity of the eyepiece might well not be attained, but the large residual suggested that the goodness of fit of (1) should be investigated in a more detailed way. We therefore made an examination of the signs of the residuals (Jeffreys, 1961). 
Suppose we have a series of $n$ measurements to which a polynomial equation containing $m$ parameters has been fitted, and suppose that the probability of a change of sign between successive residuals is $z$. There are $n-1$ possible changes of sign, and $m$ are ensured by the fitting of an $m$-parameter curve. Thus the effectively variable number of changes of sign will be $n-m-1$, and the probability of finding actually $s$ changes will be given by the binomial term

$$
\left(\begin{array}{c}
n-m-1 \\
s-m
\end{array}\right) z^{s-m}(1-z)^{n-s-1}
$$

-provided that the assumed equation is a true representation of the law underlying

Table 2. Mean, standard deviation (s.D.) and range of generation time ( $\tau$ ), length at inception $\left(x_{i}\right)$, length at termination $\left(x_{t}\right)$ and mean growth rate $(\kappa)$; deviation from linearity $(\Delta)$ and corrected ratios $\left(p_{I}, p_{o}\right)$ of $x_{i}$ (daughter) to $x_{t}$ (mother). Unit of time : 1 min.; unit of length: $1 \mu$; number of observations: $n$

\begin{tabular}{|c|c|c|c|c|c|c|c|c|}
\hline \multicolumn{2}{|c|}{ Series } & $\tau$ & $x_{i}$ & $x_{t}$ & $10^{2} \kappa$ & $10^{2} \Delta$ & $p_{I}$ & $p_{O}$ \\
\hline \multirow[t]{5}{*}{ Sm 1} & Mean & $22 \cdot 4$ & $2 \cdot 86$ & $5 \cdot 59$ & $3 \cdot 02$ & - & $0 \cdot 491$ & 0.509 \\
\hline & S.D. & 6.51 & 0.47 & 0.85 & $0 \cdot 36$ & - & - & - \\
\hline & Bange & $\int 13$ & 2.04 & $4 \cdot 29$ & 1.92 & -13.1 & 0.431 & 0.491 \\
\hline & Kange & $\{42$ & $4 \cdot 42$ & $8 \cdot 77$ & $\mathbf{3} \cdot \mathbf{4 4}$ & $+14 \cdot 4$ & 0.545 & 0.543 \\
\hline & $n$ & 20 & 23 & 27 & 19 & 19 & 12 & 8 \\
\hline \multirow[t]{5}{*}{$\operatorname{Pm} 1$} & Mean & $27 \cdot 1$ & 4.53 & $8 \cdot 79$ & $2 \cdot 35$ & 一 & $0 \cdot 499$ & 0.501 \\
\hline & S.D. & $5 \cdot 72$ & 0.67 & $1 \cdot 15$ & 0.20 & - & - & - \\
\hline & & 20 & $3 \cdot 36$ & $6 \cdot 44$ & 1.93 & $-4 \cdot 2$ & 0.470 & 0.451 \\
\hline & Range & $\{41$ & $6 \cdot 30$ & $11 \cdot 87$ & $2 \cdot 69$ & $+11 \cdot 7$ & 0.532 & 0.549 \\
\hline & $n$ & 27 & 38 & 38 & 26 & 26 & 35 & 30 \\
\hline \multirow[t]{5}{*}{ Ec 1} & Mean & $25 \cdot 8$ & $3 \cdot 36$ & $6 \cdot 67$ & $2 \cdot 64$ & - & 0.494 & 0.506 \\
\hline & S.D. & $3 \cdot 27$ & 0.33 & $0 \cdot 61$ & $0 \cdot 17$ & - & - & - \\
\hline & D & 21 & $2 \cdot 94$ & $5 \cdot 54$ & $2 \cdot 37$ & -5.0 & 0.456 & 0.461 \\
\hline & Range & $\left\{\begin{array}{l}33 \\
33\end{array}\right.$ & $4 \cdot 45$ & $7 \cdot 92$ & $2 \cdot 90$ & $+13 \cdot 2$ & 0.531 & 0.575 \\
\hline & $n$ & 20 & 23 & 28 & 18 & 18 & 11 & 9 \\
\hline \multirow[t]{5}{*}{ Ec 2} & Mean & $31 \cdot 1$ & 4.54 & $8 \cdot 99$ & $2 \cdot 19$ & - & 0.499 & 0.504 \\
\hline & S.D. & $5 \cdot 91$ & 1.08 & $2 \cdot 28$ & 0.29 & - & - & - \\
\hline & Panme & $\int 22$ & $3 \cdot 17$ & $5 \cdot 89$ & $1 \cdot 37$ & -6.4 & 0.413 & 0.434 \\
\hline & Range & $\{45$ & $7 \cdot 51$ & $16 \cdot 35$ & $2 \cdot 68$ & $+13 \cdot 2$ & $0 \cdot 629$ & 0.594 \\
\hline & $n$ & 25 & 40 & 42 & 25 & 25 & 30 & 26 \\
\hline \multirow[t]{5}{*}{$\mathbf{P a} 1$} & Mean & $32 \cdot 7$ & $\mathbf{3 \cdot 2 6}$ & $6 \cdot 32$ & $2 \cdot 04$ & - & 0.493 & 0.508 \\
\hline & S.D. & $4 \cdot 64$ & $0 \cdot 40$ & 0.76 & $0 \cdot 19$ & - & - & - \\
\hline & Dona & $\int 24$ & $2 \cdot 48$ & $5 \cdot 11$ & $1 \cdot 74$ & $-11 \cdot 3$ & 0.453 & 0.474 \\
\hline & Range & $\{40$ & $4 \cdot 04$ & $7 \cdot 89$ & $2 \cdot 47$ & +13.0 & 0.535 & 0.552 \\
\hline & $n$ & 30 & 31 & 33 & 30 & 30 & 5 & 16 \\
\hline \multirow[t]{5}{*}{$\operatorname{Pa} 2$} & Mean & $\mathbf{5 2} \cdot 4$ & $2 \cdot 82$ & $5 \cdot 53$ & $1 \cdot 18$ & 一 & $0 \cdot 494$ & 0.507 \\
\hline & s.D. & $11 \cdot 23$ & $0 \cdot 47$ & 0.73 & 0.21 & - & - & - \\
\hline & 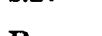 & $(39$ & 1.86 & $3 \cdot 76$ & 0.75 & $-11 \cdot 3$ & 0.4110 & 0.450 \\
\hline & Range & $\left\{\begin{array}{l}83 \\
8\end{array}\right.$ & $3 \cdot 45$ & $6 \cdot 94$ & $1 \cdot 56$ & $+11 \cdot 1$ & 0.550 & 0.611 \\
\hline & $n$ & 20 & 38 & $\mathbf{3 3}$ & 20 & 20 & 21 & 16 \\
\hline \multirow{2}{*}{ Sm 1} & & $\int-$ & $2 \cdot 04$ & 4.29 & - & - & - & 一 \\
\hline & Range & $\{-$ & $3 \cdot 51$ & $6 \cdot 82$ & - & - & - & - \\
\hline \multicolumn{9}{|c|}{ omitting 1 fission } \\
\hline \multirow{2}{*}{ Ec 2} & Range & $\{-$ & $\mathbf{3} \cdot 39$ & $6 \cdot 69$ & 一 & - & - & - \\
\hline & \multicolumn{8}{|c|}{ omitting 4 fissions } \\
\hline \multicolumn{3}{|l|}{8} & & & & & \multicolumn{2}{|c|}{ G. Microb. $\mathrm{xxxIX}$} \\
\hline
\end{tabular}




\section{F. P. Errington, E. O. Powell and N. Thompson}

the measurements. The expected value of $s$ is then $m+(n-m-1) z$. We formed a $\chi^{2}$ for each fitting of (1):

$$
\chi^{2}=\frac{\{(s-m)-(n-m-1) z\}^{2}}{(n-m-1) z}+\frac{\{(n-s-1)-(n-m-1)(1-z)\}^{2}}{(n-m-1)(1-z)}
$$

(1 degree of freedom). As Powell \& Errington (1963b) explained, the method of measurement we adopted resulted in association between successive residuals, and the appropriate value of $z$ was consequently about $1 / 3 ; m$ was 3 , whence

$$
\chi^{2}=(3 s-n-5)^{2} / 2(n-4) .
$$

Of 163 curves thus tested, 29 gave a $\chi^{2}$ exceeding the 0.05 probability limit; in only 2 of the 29 did $s$ exceed its expectation. The sum of $\chi^{2}$ for the 163 was 326 . We conclude that (1) is usually a satisfactory representation of the law of growth, but that

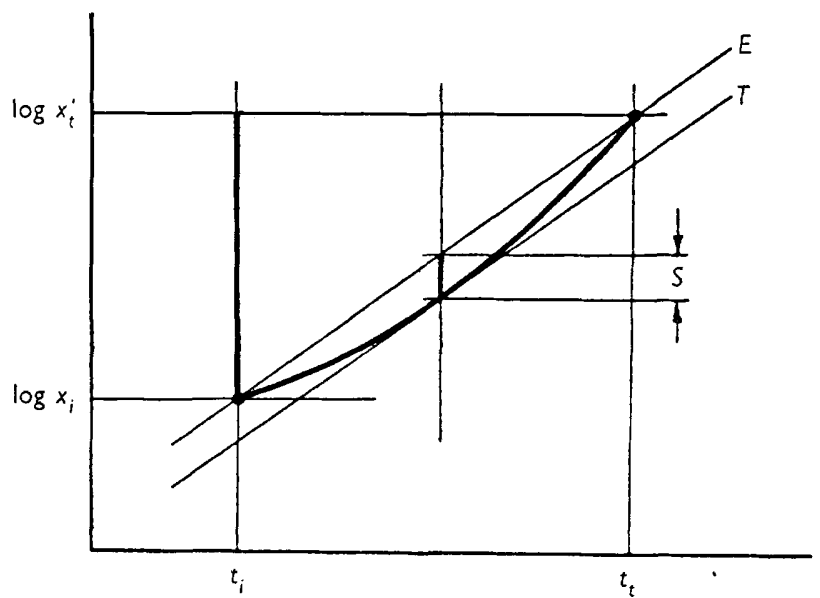

Fig. 4

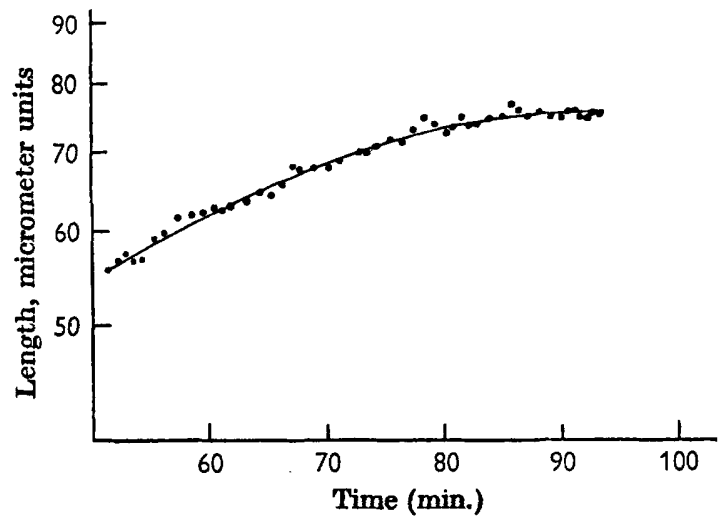

Fig. 5

Fig. 4. The definition of $\Delta$. The heavy curved line represents a typical growth curve. Through its end points $\left(t_{i}, \log x_{i}\right),\left(t_{t}, \log x_{t}^{\prime}\right)$ draw a straight line $E$. Draw $T$ parallel to $E$ and tangent to the curve. The vertical distance between $T$ and $E$, measured upwards from $T$, is $S$. Then

$$
\Delta=\frac{S}{\log \left(x_{t}^{\prime} \mid x_{i}\right)}
$$

Fig. 5. Early growth of a non-viable organism of Pseudomonas aeruginosa (series Pa 1).

runs of residuals of the same sign are improbably frequent. The fluctuations in growth rate differ from one organism to another, and cannot be assimilated to a quite simple formula. This may not be so for the law of growth in volume, to which length measurements give only an approximation.

The crude numerical results are summarized in Table 2. Instead of recording the coefficients of equation (1) (of which $\alpha$ and $\beta$ are not independent of the time origin) we have characterized the individual growth curves by the two quantities $\kappa$ and $\Delta$. Of these, $\kappa$ is the mean growth rate over the life-span of the organism:

$$
\kappa=\frac{\log \left(x_{t}^{\prime} \mid x_{i}\right)}{\tau} \text {. }
$$

Here the $x_{i}$ and $x_{t}^{\prime}$ belong to the same organism, not the same fission, and they were 
estimated from the fitted curves (equation (2)). The definition of $\Delta$ is given in Fig. 4; it appears unnecessarily complicated, but $\Delta$ gives a more immediate and appealing impression than does $\gamma$ of the degree of curvature over the life-span of the organism. For equation (1)

$$
\Delta=\gamma \tau / 4 \kappa
$$

and so it has the same sign as $\gamma$. In Fig. 2, the curve of accelerated growth in length has $\Delta=+0.028$.

In the course of the experiments we happened upon two organisms which turned out to be non-viable. The growth of one of them is illustrated in Fig. 5. Although its size did increase during the earlier stages of observation, the initial growth rate was only about $0.014 \mathrm{~min}^{-1}$ - smaller than the mean rate for any other organism in the series. The other was the daughter of a mother notable for having much the smallest $x_{t}$ in the series (Ec 2).

\section{Some general features of the family tree}

To permit synoptic examination we made lists of the following quantities (where they were known) belonging to each organism:

(i) The position symbol, $I$ or $O$.

(ii) The generation time, $\tau$.

(iii) The length at inception and termination, estimated from the fitted growth curves.

(iv) The mean growth rate of the individual, $\kappa$.

(v) The sign of the coefficient $\gamma$ in equation (1).

(vi) A quantity $q$ relating the length of the organism at inception to the length of its mother at termination:

$$
q=2 x_{i}-x_{t} \text { (mother). }
$$

(vii) The ratio, $p$, of length of daughter to length of mother. Actually a small correction (discussed below, p. 116) was deducted from the estimated lengths before forming the $p$. The correction did not alter the ranking of the $p$ in order of magnitude.

(viii) The sign of $\left(x_{t}^{\prime} / x_{i}-2\right), x_{i}$ and $x_{t}$ 'belonging to the same organism. This quantity is positive or negative according as the organism increases in size by more or less than a factor of 2 over its life-span.

\section{Table 3. Serratia marcescens, series $\boldsymbol{S m} 1$}

Association between position symbol and mean growth rate. $V=+\mathbf{0 \cdot 3 8 7}$.

$\begin{array}{cccc} & \begin{array}{c}\kappa> \\ \text { sample } \\ \text { median }\end{array} & \begin{array}{c}\kappa< \\ \text { sample } \\ \text { median }\end{array} & \begin{array}{c}\text { Row } \\ \text { total }\end{array} \\ I & 6 \frac{1}{2} & 3 \frac{1}{2} & 10 \\ \text { Column total } & 1 \frac{1}{2} & 4 \frac{1}{2} & 6 \\ & 8 & 8 & 16\end{array}$

From pairs of some of these quantities we computed, for each series of experiments, Yule's coefficient of absolute association, $V$ (Kendall, 1947). In order to form the classes of a $2 \times 2$ contingency table, the range of metrical quantities was dichotomized at the sample median (Table 3 gives an example). Our sample sizes were too small to justify the computation of a large number of product-moment 


\section{F. P. Errington, E. O. Powell and N. Thompson}

correlations, and we expected to detect only the cruder and more regular features of the growth pattern. Many of the individual $V$ were statistically non-significant, but the combined significance of the $V$ of any one kind was tested by forming the $\chi^{2}$ from the class totals in the usual way. In the first place we accepted as suggestive only those $V$ which had the same sign in every series of experiments.

We found the following to be true of every series:

(a) Position symbol $I$ is associated with $p<\frac{1}{2}$. In pairs of sisters, the inner organism is usually the smaller at inception. $\chi^{2}=0.9$. Also, in 26 pairs of sisters of which both members were measured, the inner was the smaller in 19 instances. $\chi^{2}=5 \cdot 5$.

(b) Position symbol $I$ is associated with large $\kappa$. In pairs of sisters, the inner usually grows the faster. $\chi^{2}=6 \cdot 4$.

(c) Large $\kappa$ is associated with $p<\frac{1}{2}$. In pairs of sisters, the smaller usually grows the faster. $\chi^{2}=7 \cdot 1$.

Although the $\chi^{2}$ is small, the first part of $(a)$ is significant at the 0.05 probability level by virtue of the uniform sign of the $V$ alone, and it is supported by the second part. The other two statements have much greater weight because of the large $\chi^{2}$. The following two generalizations are less striking because only five of the six $V$ were of the same sign; the $\chi^{2}$ are both significant at the 0.05 level:

(d) Position symbol $I$ is associated with a positive value of $\left(x_{t}^{\prime} / x_{i}-2\right)$. In pairs of sisters, the inner usually increases in size by more, the outer by less, than a factor of 2. $\chi^{2}=4 \cdot 4$.

(e) A positive value of $\left(x_{t}^{\prime} \mid x_{i}-2\right)$ is associated with $p<\frac{1}{2}$. In pairs of sisters, the smaller usually increases in size by more, the larger by less, than a factor of 2. $\chi^{2}=4 \cdot 1$.

If two of $V(x, y), V(y, z), V(z, x)$ are of like sign, it does not follow that the third is positive; the triads $\{(a)(b)(c)\},\{(a)(d)(e)\}$ do not include a redundant statement. We thus have evidence for two interesting properties of the growth pattern. First, fission is unsymmetrical in respect of size at inception and mean growth rate; the lack of symmetry is not random but is systematically related to the position symbol. Secondly, there appears to be some regulation of the individual growth rate which partially compensates for inequality in the size of sisters at inception. The regulation is imperfect; within each series of experiments we found the generation time still to be negatively associated with absolute size at inception. On the other hand, the excess or deficit in $\kappa$ is more than enough to offset the tendency of inner organisms to have shorter generation times than outer organisms (Powell \& Errington, 1963a).

\section{Fission into organisms of unequal size}

The use of the quantity $q$ (equation 5) as a measure of the relativesi zes of mother and daughter at fission was suggested by Powell \& Errington's (1963b) experience that the estimated $x_{i}$ of sisters were together greater than the $x_{t}$ of the mother. In our experiments, the number of inner and outer organisms measured were not usually equal, but we also found that in every series, the mean $q$ associated with inner $\left(\bar{q}_{I}\right)$ and outer $\left(\bar{q}_{o}\right)$ organisms were together greater than 0 . The excess can be ascribed to two causes: an unknown systematic error of the micrometer and a systematic increase due to the rounding of the ends of the organisms at fission, 
making them for a given volume longer than cylinders of the same diameter bounded by plane ends. We had no means of estimating these errors separately, and we therefore computed for each series a length correction $\delta$ given by

$$
2 \delta=\bar{q}_{I}+\bar{q}_{O}
$$

which, since it was small $(0 \cdot 05-0 \cdot 15 \mu)$, we assumed to apply throughout the series.

In the Koch \& Schaechter hypothesis it is more convenient to use the ratio $p$ instead of $q$ :

$$
p=\frac{\left.x_{i} \text { (daughter }\right)}{x_{t} \text { (mother) }} .
$$

The frequency function (say $k(p)$ ) of $p$ and its parameters play a large part in quantitative inferences from the hypothesis. The dispersion of $p$ is in practice quite small, and we considered it desirable to attempt a more accurate estimate of the volume ratio than that given by (6). We therefore worked with $p$ based on corrected lengths, i.e. with

$$
p=\frac{x_{i}-\delta}{x_{t}-\delta} .
$$

Table 2 shows that the mean values $\bar{p}_{I}, \bar{p}_{O}$ differed systematically, but their sum was very nearly unity in each series. The separate distributions of $p_{I}$ and $p_{O}$ must be unsymmetrical, each a mirror image of the other in the line $p_{I}=p_{O}=\frac{1}{2}$. But in a growing culture equal numbers of inner and outer organisms are formed and the distribution $k(p)$ of $p$ generally is symmetrical.

Table 4. Sample statistics of the distribution of the corrected ratios $p$, the correlation $\rho\left(x_{t}, \theta\right)$ (equation (7)), and the correlation between the logarithms of $p$ and terminal size at succeeding fission. Number of observations in parentheses

$\begin{array}{lcccc}\text { Series } & -\mu_{1}^{\prime}(\log p) & 10^{3} \mu_{2}(\log p) & \rho\left(x_{t}, \theta\right) & \rho\left(\log p, \log x_{t}^{\prime}\right) \\ \text { Sm 1 } & 0.69471 & 3 \cdot 210 & +0.465(22) & -0.029(17) \\ \text { Pm 1 } & 0.69419 & 2 \cdot 098 & -0.212(38) & +0.354(26) \\ \text { Ec 1 } & 0.69450 & 2 \cdot 771 & -0.170(23) & +0.062(18) \\ \text { Ec 2 } & 0.69623 & 6 \cdot 216 & +0.023(40) & -0.195(25) \\ \text { Pa 1 } & 0.69439 & 2 \cdot 482 & +0 \cdot 113(22) & -0.067(20) \\ \text { Pa 2 } & 0.69626 & 6 \cdot 290 & +0.199(38) & +0 \cdot 158(20)\end{array}$

For purposes of record, the first and second sample moments of $\log p$ are listed in Table 4.

In its simplified form, the Koch \& Schaechter hypothesis requires the distribution of $p$ to be the same for all $x_{t}$ (Powell, 1964). We might expect to detect a trend in the dispersion of $p$ by forming the correlation coefficient between $x_{t}$ and $\left|p-\frac{1}{2}\right|$. The distribution of $\left|p-\frac{1}{2}\right|$, however, is J-shaped and highly skew, and the customary significance test cannot be legitimately applied to $\rho\left(x_{t},\left|p-\frac{1}{2}\right|\right)$. Graphical experiment with our data showed that the variate

$$
\theta,=\log \left\{\frac{1}{2\left|p-\frac{1}{2}\right|}-1\right\},
$$

(whose range is $-\infty$ to $+\infty$ ) was much more nearly normal and not significantly skew. Therefore the six series of $p$ were converted to $\theta$ and the correlation coefficients $\rho\left(x_{t}, \theta\right)$ determined. Of the six $\rho$ (Table 4 ) only the first differs from zero 
at the $\mathbf{0 . 0 5}$ probability level-not an unlikely event among six examples-and not all are of the same sign. So far, we can conclude that the distribution of $p$ is roughly the same for all $x_{t}$.

Another requirement of the hypothesis is that the size of an organism at termination $\left(x_{t}^{\prime}\right)$ should be independent of the $p$ associated with its inception; in particular, that $\operatorname{cov}\left(\log p, \log x^{\prime}\right)=0$ (Powell, 1964). We therefore computed also the $\rho\left(\log p, \log x_{t}^{\prime}\right)$ and found none to be significant at the 0.05 probability level; three were positive and three negative (Table 4).

\section{The dispersion of individual mean growth rates}

The dispersions of generation time and individual mean growth rate are compared in Table 5. It will be seen that the coefficient of variation $c(\kappa)$ of $\kappa$ is always less, but not always much less, than that of $\tau$. The fact that the experimental $\kappa$ are systematically associated with $p$ and with position symbol makes it almost certain that the dispersion of $\kappa$ is real, but we may ask how much of the sample variances

Table 5. Comparison of the variances $\left(\sigma^{2}\right)$ and squared coefficients of variation $\left(c^{2}\right)$ of generation time $(\tau)$ and mean growth rate $(\kappa)$

$\begin{array}{lrccl}\text { Series } & \sigma^{2}(\tau) & 10^{2} c^{2}(\tau) & 10^{6} \sigma^{2}(\kappa) & 10^{2} c^{2}(\kappa) \\ \text { Sm 1 } & 42 \cdot 34 & 8 \cdot 477 & 12 \cdot 89 & 1 \cdot 417 \\ \text { Pm 1 } & 32 \cdot 75 & 4 \cdot 443 & 4 \cdot 182 & 0 \cdot 7547 \\ \text { Ec 1 } & 10 \cdot 69 & 1 \cdot 607 & 2 \cdot 944 & 0 \cdot 4226 \\ \text { Ec 2 } & 34 \cdot 94 & 3 \cdot 608 & \mathbf{8} \cdot 802 & 1 \cdot 836 \\ \text { Pa 1 } & 21 \cdot 54 & 2 \cdot 019 & 3 \cdot 435 & 0 \cdot 8256 \\ \text { Pa 2 } & 126 \cdot 04 & 4 \cdot 590 & 4 \cdot 247 & \mathbf{3} \cdot 029\end{array}$

may have been contributed by simple errors of measurement. From the definition of $\kappa$ (equation (3)) it can be seen that estimates of it can be affected both by errors in measuring length and by errors in estimating times of fission. Temporarily, we shall use subscripts $x, t$ to distinguish components of variance due to these errors.

First, $\kappa$ is hardly affected by errors in estimating times of fission. From (2) and (3)

$$
\begin{aligned}
\kappa & =\left\{\left(\alpha+\beta t_{t}+\gamma t_{t}^{2}\right)-\left(\alpha+\beta t_{i}+\gamma t_{i}^{2}\right)\right\} /\left(t_{t}-t_{i}\right) \\
& =\beta+\gamma\left(t_{t}+t_{i}\right) .
\end{aligned}
$$

The uncertainties, say $\delta t$, in $t_{t}$ and $t_{i}$ are alike and independent, hence the uncertainty in $\kappa$ is

$$
\delta \kappa=\sqrt{ } 2 \gamma \delta t
$$

and the expectation of $(\delta \kappa)^{2}$ namely $\sigma_{t}^{2}(\kappa)$, is

$$
\overline{2 \gamma^{2}}(\delta t)^{2} \text {. }
$$

In series Ec 1, for which $\sigma^{2}(\kappa)$ was smallest, $\overline{\gamma^{2}}$ was $3 \cdot 2 \times 10^{-8}$, giving

$$
\sigma_{t}^{2}(\kappa)=\left(6 \cdot 4 \times 10^{-8}\right)(\delta t)^{2} .
$$

It is difficult to obtain an estimate of $\delta t$ except by comparing the judgement of several observers about the occurrence of particular fissions. Experience suggests $\delta t \bumpeq 1 \mathrm{~min}$. for fast growing cultures, and then

$$
\sigma_{t}^{2}(\kappa)=6 \cdot 4 \times 10^{-8}
$$

This is quite negligible in comparison with $\sigma^{2}(\kappa)$. 
Secondly, we can obtain a rough but simple estimate of $\sigma_{x}^{2}(\kappa)$ by neglecting the small curvature and treating the length measurements as uniformly spaced in time (they were nearly so). If the uncertainty $\delta \log x$ in $\log x$ were constant, we should then have (see e.g. Jeffreys, 1961)

$$
(\delta \kappa)^{2}=\frac{12}{n \tau^{2}}(\delta \log x)^{2},
$$

where $n$ is the number of co-ordinate pairs $t_{r}, x_{r}$. Now $\delta \log x \bumpeq \delta x / x$, and for any one organism $x$ varies from $x_{i}$ to $x_{t}^{\prime}$. An upper limit to $\sigma_{x}^{2}(\kappa)$ is therefore given by putting minimum values for the variable terms in the denominator of (8). In series Ec 1 these were $n=23, \tau=21, x_{i}=2.9 \mu$, and

$$
\sigma_{x}^{2}(\kappa)<\left(1.4 \times 10^{-4}\right)(\delta x)^{2} .
$$

Under the best conditions, $\delta x$ is about $0.03 \mu$, but since the $\kappa$ were determined through the application of equation (3), it is proper to take as $\delta x$ the r.m.s. residual from that equation, namely $0.076 \mu$. Then

$$
\sigma_{x}^{2}(\kappa)<8.1 \times 10^{-7} .
$$

In series Ec 1, therefore, not more than a quarter of the observed variance of $\kappa$ can be ascribed to errors of measurement, and in the other series the fraction is still smaller.

There remains the possibility that $\sigma^{2}(\kappa)$ is inflated by imperfect replication of the experimental conditions. We therefore carried out two-way analyses of variance for within- and between-experiment components of variance. Because the sample sizes were small, and because we might expect $\tau$ and $\kappa$ to be affected to a similar extent (though in opposite senses) by imperfect replication, the analyses were carried out on the $\tau$ as well as the $\kappa$. Only one of the 12 analyses (for $\kappa$ in Ec 2) yielded an $\boldsymbol{F}$-ratio above the 0.05 probability limit, and six of the ratios were less than unity. Previous, less happy, experience suggests that this result was no more than fortunate; but we are left with the conclusion that $\sigma^{2}(\kappa)$ cannot for the most part be attributed to recognizable experimental error.

\section{Statistical independence of size of organism at termination}

According to the Koch \& Schaechter hypothesis, if an organism has attained a size $x$, its chance of dividing at the greater size $x_{t}$ is independent of its history before attaining $x$. Powell (1964) showed that the $x_{t}$ will not then be statistically independent of one another in general, but that they will be independent if the distributions of $x_{i}$ and $x_{t}$ do not overlap. It will be seen from Table 2 that Powell's condition is satisfied in Series Pm 1, Ec 1, Pa 1 and Pa 2: the extremes of $x_{i}$ stand in a ratio less than $2: 1$; the same is true of $x_{t}$; the largest $x_{t}$ is less than four times the smallest $x_{i}$; the largest $x_{i}$ is less than the smallest $x_{t}$; no $\tau$ is greater than twice the mean.

But Powell did not notice that if by chance or selection we obtain a group of organisms in which the sample $x_{i}$ and $x_{t}$ obey his condition, the $x_{t}$ will be statistically independent of one another, even if in the whole culture the condition is not metprovided the Koch \& Schaechter hypothesis is true. We can make use of our data in series Sm 1 and Ec 2 by omitting the $x_{i}$ and $x_{t}$ associated with 5 fissions, and so reducing the range sufficiently (Table 2 ). In this section, therefore, the outlying 
$x_{i}$ and $x_{t}$ have been omitted from the calculations for these two series. Sample values of two correlation coefficients

and

$$
\rho\left(X_{t t}\right) \equiv \rho\left(\log x_{t}, \log x_{t}^{\prime}\right)
$$

are given in Table 6; here $x_{t}, x_{t}^{\prime}$ distinguish sizes associated with successive fissions (and therefore with mother and daughter), while $x_{i}, x_{t}^{\prime}$ distinguish sizes associated with the same organism. All the $\rho$ are positive and all but one significantly different from zero at the 0.05 probability level.

Table 6. Sample values of the crude $\left(\sigma^{2}\right)$ and inter-experiment $\left(\sigma_{E}^{2}\right)$ variances of $\log x_{i}$ and $\log x_{t} ;$ the ratios $\boldsymbol{R}_{t t}=\sigma_{E}^{2}\left(\log x_{t}\right) / \sigma^{2}\left(\log x_{t}\right)$ and $\boldsymbol{R}_{i t}=\sigma_{E}\left(\log x_{i}\right) \sigma_{E}\left(\log x_{t}\right) /$ $\sigma\left(\log x_{i}\right) \sigma\left(\log x_{t}\right)$ and the correlation coefficients, $\rho\left(X_{t t}\right), \rho\left(X_{i t}\right)$ between $\log x_{t}$ and $\log x_{t}^{\prime}$, $\log x_{i}$ and $\log x_{t}^{\prime}$

\begin{tabular}{|c|c|c|c|c|c|c|c|c|}
\hline Series & $10^{2} \sigma^{2}\left(\log x_{i}\right)$ & $10^{2} \sigma_{E}^{2}\left(\log x_{i}\right)$ & $10^{2} \sigma^{2}\left(\log x_{t}\right)$ & $10^{2} \sigma_{E}^{2}\left(\log x_{t}\right)$ & $\boldsymbol{R}_{t t}$ & $\rho\left(\boldsymbol{X}_{t t}\right)$ & $\boldsymbol{R}_{i t}$ & $\rho\left(\boldsymbol{X}_{i t}\right)$ \\
\hline $\operatorname{Sm} 1$ & $2 \cdot 284$ & $0 \cdot 378$ & $1 \cdot 870$ & $0 \cdot 123$ & $0 \cdot 066$ & $0 \cdot 546(18)$ & $0 \cdot 104$ & $0.431(20)$ \\
\hline Pm 1 & $2 \cdot 161$ & 0 & $1 \cdot 798$ & 0 & $\mathbf{0}$ & $0.568(26)$ & 0 & $0.596(26)$ \\
\hline Ee 1 & $0 \cdot 873$ & $0 \cdot 016$ & 0.815 & $0 \cdot 119$ & $0 \cdot 146$ & $0.537(19)$ & $0 \cdot 051$ & $0.682(18)$ \\
\hline Ec 2 & $3 \cdot 546$ & $2 \cdot 053$ & $3 \cdot 572$ & $\mathbf{2} \cdot \mathbf{3 8 0}$ & 0.666 & $0.756(20)$ & $0 \cdot 621$ & $0.794(20)$ \\
\hline $\operatorname{Pa} 1$ & $1 \cdot 562$ & 0.614 & $1 \cdot 445$ & 0.775 & 0.536 & $0 \cdot 652(21)$ & 0.459 & $0.620(30)$ \\
\hline Pa 2 & $2 \cdot 115$ & 0.722 & $1 \cdot 798$ & 0.936 & 0.521 & $0.672(19)$ & 0.422 & 0.750 \\
\hline
\end{tabular}

As in all investigations of this kind, the comparison of hypothesis with observation is bedevilled by the possibility that imperfect replication of experimental conditions has inflated the variances and biased the correlations; there is the further complication that family likenesses may produce a genuine lack of homogeneity which will be interpreted as imperfect replication. The analyses of variance carried out on the $\kappa$ and $\tau$ (see previous section) seemed to show that we had been successful in reproducing the experimental conditions. But it by no means follows that the sizes of organisms are also unchanged in a replicate experiment which leaves the $\kappa$ and $\tau$ unchanged. It is logically possible, for example, to have two cultures in which the $\kappa$ and $\tau$ have identical distributions and an identical structure of inter-relations, but in one of which the sizes are a constant multiple of those in the other. In fact, two-way analyses of variance on $\log x_{i}$ and $\log x_{t}$ showed that in three series there were significant (and very large) components $\sigma_{E}^{2}\left(\log x_{i}\right), \sigma_{E}^{2}\left(\log x_{t}\right)$ of apparent interexperiment variance (Table 6).

It follows from Powell's (1958) formulae that if $\rho\left(X_{t t}\right)$ is really zero, the value to be expected as a result of inter-experiment variance is $\sigma_{E}^{2}\left(\log x_{t}\right) / \sigma^{2}\left(\log x_{t}\right),=\boldsymbol{R}_{t t}$, say; and, by simple extension of his reasoning, if $\rho\left(X_{i t}\right)$ is really zero, the value to be expected is $\sigma_{E}\left(\log x_{i}\right) \sigma_{E}\left(\log x_{t}\right) / \sigma\left(\log x_{i}\right) \sigma\left(\log x_{t}\right),=\boldsymbol{R}_{i t}$, say. All the $\rho$ in Table 6 exceed the corresponding variance ratios, and the excess is nowhere marginal, as is shown by an inverse hyperbolic tangent (z) transformation. The $\sigma, \sigma_{E}$ and $\rho$ were not based on exactly the same samples of $x_{i}$ and $x_{t}$, however, so that the statistical significance of the excess is uncertain; but we can argue from the uniformity in the sign of $\rho\left(\boldsymbol{X}_{t t}\right)-\boldsymbol{R}_{t t}$ and $\rho\left(\boldsymbol{X}_{i t}\right)-\boldsymbol{R}_{i t}$. Since the $\rho$ were in large part calculated from $x_{i}$ and $x_{t}$ belonging to the same fissions, and since $x_{i} / x_{t}$ never differs much from $\frac{1}{2}$, the two sets of $\rho$ are not independent; they have the weight not of twelve but only 
of rather more than six independent estimates. The probability to be attached to the uniformity in sign of the $\rho-R$ is thus something less than $2^{-5}(=1 / 32)$, but not as little as $2^{-11}(=1 / 2048)$.

We can also show by a non-parametric test, independent of errors of replication, that the $x_{t}$ at successive fissions are associated. Though it can tell us less about the intensity of the association, it gives a result of higher statistical significance than the foregoing discussion of the $\rho$. We can say that if $x_{m}$ is the median terminal size in an individual experiment, then if $x_{t}, x_{t}^{\prime}$ are successive terminal sizes also belonging to the experiment, the product $\left(x_{t}-x_{m}\right)\left(x_{t}^{\prime}-x_{m}\right)$ will be positive or negative according as $x_{t}$ and $x_{t}^{\prime}$ lie on the same or opposite sides of $x_{m}$, and the probabilities of these events will be equal if there is no association between $x_{t}$ and $x_{t}^{\prime}$. The frequencies of positive and negative products are collected together in Table 7. In every series

Table 7. The sign of the product $\left(x_{t}-x_{m}\right)\left(x_{t}^{\prime}-x_{m}\right)$, where $x_{t}, x_{t}^{\prime}$ are the terminal sizes at successive fissions, and $x_{m}$ is the experiment sample median

\begin{tabular}{lccc} 
& \multicolumn{2}{c}{$\begin{array}{c}\text { Frequency with which } \\
\left(x_{t}-x_{m}\right)\left(x_{t}^{\prime}-x_{m}\right)\end{array}$} \\
Series & $\overbrace{\text { +ve }}$ & 0 & - ve \\
Sm 1 & 9 & 1 & 7 \\
Pm 1 & 16 & 2 & 8 \\
Ec 1 & 10 & 4 & 5 \\
Ec 2 & 12 & 5 & 4 \\
Pa 1 & 13 & 3 & 5 \\
Pa 2 & 7 & 8 & 6 \\
Sum & 67 & - & 35 \\
& \multicolumn{3}{|c}{102}
\end{tabular}

positive signs are in excess, and the total frequencies differ by 32 , more than six times the standard deviation $\sqrt{(102) / 2}$ on the hypothesis of independence: probability less than $0 \cdot 003$. This estimate of significance is really conservative; the use of sample medians instead of the unknown true medians in the test biases the sign frequencies towards equality.

We thus have fairly strong evidence that the size of an organism at termination is not independent of the circumstances of its inception.

\section{CONCLUSION}

It now seems that both of the major postulates of the Koch \& Schaechter hypothesis are wrong. But we have not yet shown that they are quantitatively so far wrong as to make the hypothesis valueless; our experiments are exploratory only. We expect the hypothesis to remain as a framework into which refinements can be introduced, and to be more adaptable in this respect than older hypotheses about the pattern of generation times.

According to the original hypothesis we have (Powell, 1964)

and approximately

$$
\nu^{2} \sigma^{2}(\tau)=2 \sigma^{2}\left(\log x_{t}\right)+\sigma^{2}(\log p)
$$

$$
c^{2}(\tau)=4 c^{2}\left(x_{t}\right)
$$


But if we admit that $\kappa$ may be dispersed and $\rho\left(X_{t t}\right)$ not zero, we must now write (from (3))

$$
\begin{aligned}
\sigma^{2}(\kappa \tau) & =2 \sigma^{2}\left(\log x_{t}\right)-2 \operatorname{cov}\left(\log x_{t}, \log x_{t}^{\prime}\right)+\sigma^{2}(\log p) \\
& =2\left\{1-\rho\left(X_{t t}\right)\right\} \sigma^{2}\left(\log x_{t}\right)+\sigma^{2}(\log p) .
\end{aligned}
$$

Neglecting $\sigma^{2}(\log p)$ as small compared with $\sigma^{2}\left(\log x_{t}\right)$, and $c^{2}(\kappa)$ as small compared with $c^{2}(\tau)$, we now have instead of the approximation (9)

$$
c^{2}(\tau)=4\left\{1-\rho\left(X_{t t}\right)\right\} c^{2}\left(x_{t}\right):
$$

if $\rho\left(X_{t t}\right)$ is positive, $c(\tau)$ will be less than twice $c\left(x_{t}\right)$. But if in (10) we substitute sample values such that the $\tau$ on the left belong to the same organisms as the $x_{t}$ on the right, we necessarily obtain approximate agreement, and the comparison adds nothing to a discussion of the significance of sample $\rho$ computed directly from the data. (We can obtain a useful comparison, however, by taking the $c^{2}(\tau)$ from one sample of organisms and the $c^{2}\left(x_{t}\right)$ and $\rho\left(X_{t t}\right)$ from an independent sample; our own data were not numerous enough to sustain this subdivision.) On the other hand, Schaechter et al. (1962) do not record sample values of $\rho\left(X_{t t}\right)$, and we can apply (10) to their published data in order to obtain at least a rough impression of the magnitude of $\rho\left(X_{t t}\right)$. In six of seven experiments for which Schaechter et al. record both $c(\tau)$ and $c\left(x_{t}\right), c(\tau)$ is less than $2 c\left(x_{t}\right)$ (in two of them, much less); thus $\rho\left(X_{t t}\right)$ seems to be on the whole positive (Table 8). If it were zero, we might expect a tendency for $c(\tau)$ to exceed $2 c\left(x_{t}\right)$ because of the neglected variances $\sigma^{2}(\log p)$ and $\sigma^{2}(\kappa)$.

Table 8. Coefficients of variation of $x_{t}$ and $\tau$ in seven experiments from Schaechter et al. (1962). Estimates of $\rho\left(X_{t t}\right)$ obtained from $c\left(x_{t}\right)$ and $c(\tau)$ by means of equation (10)

$\begin{array}{cccr}\text { Expt. no. } & c\left(x_{t}\right) & c(\tau) & \rho\left(\boldsymbol{X}_{t t}\right) \\ \text { A-1 } & 0.085 & 0.176 & -0.07 \\ \text { A-3 } & 0.089 & 0.137 & +0.41 \\ \text { B-4 } & 0.099 & 0.169 & +0.27 \\ \text { B-5 } & 0.107 & 0.152 & +0.50 \\ \text { F-1 } & 0.118 & 0.228 & +0.07 \\ \text { F-2 } & 0.130 & 0.144 & +0.69 \\ \text { F-3 } & 0.090 & 0.179 & +0.01\end{array}$

We cannot be surprised if $\rho\left(X_{t t}\right)$ should turn out to be always appreciably positive; we know already that the pattern of growth exhibits hereditary elements extending beyond those implied by the Koch \& Schaechter hypothesis, as witness the correlations between the generation times of cousins and second cousins.

The more striking outcome of our experiments is the indication that fission is systematically unsymmetrical in several respects. Powell \& Errington (1963a) were able to detect an ordered difference in the generation times of sisters only by working with thousands of observations. The ordered differences in $\kappa$ and $p$ are detectable at about the same level of significance in less than 200 observations; they are too large to be at once dismissed as trivial consequences of the cells' mechanical structure (though they may yet be so). The manner in which the Gramnegative cell-wall is laid down is not yet settled, and may differ from one species to another; it is almost certainly more complex than Powell \& Errington supposed (Chung, Hawirko \& Isaac, 1964). While we are dubious about Chung, Hawirko \& Isaac's interpretation in terms of cross-walls and multicellular organisms, it is clear 
that the cell-wall structure of Escherichia coli at least is unsymmetrical at inception, and hence that at termination the daughters may exhibit an ordered difference in properties. The banded wall structure which Chung, Hawirko \& Isaac demonstrate may well correspond to such fluctuations in individual growth rate as would account for the failure of our equation (1) to represent the growth accurately enough.

We are indebted to L. J. Capstick and to S. Peto and his staff for much computational work.

\section{REFERENCES}

Chung, K. L., Hawirko, R. Z. \& IsaAc, P. K. (1964). Cell wall replication. II. Cell wall growth and cross wall formation of Escherichia coli and Streptococcus faecalis. Can. J. Microbiol. 10, 473.

Collins, J. F. \& Richmond, M. H. (1962). Rate of growth of Bacillus cereus between divisions. J. gen. Microbiol. 28, 15.

Dyson, J. (1959). An image-splitting microscope for accurate measurement. Nature, Lond. 184, 1561.

Dyson, J. (1960). Precise measurement by image-splitting. J. opt. Soc. Amer. 50, 754.

Jefrreys, H. (1961). Theory of Probability, 3rd edition. Oxford University Press. (See chap. 5.)

Kendall, M. G. (1947). The Advanced Theory of Statistics, 3rd edition. London: Charles Griffin and Co.

Koch, A. L. \& Schaechter, M. (1962). A model for statistics of the cell division process. J. gen. Microbiol. 29, 435.

PowELL, E. O. (1958). An outline of the pattern of bacterial generation times. J. gen. Microbiol. 18, 382.

Powell, E. O. (1964). A note on Koch and Schaechter's hypothesis about the growth and fission of bacteria. J. gen. Microbiol. 37, 231.

Powell, E. O. \& Errington, F. P. (1963a). Generation times of individual bacteria: some corroborative measurements. J. gen. Microbiol. 31, 315.

Powell, E. O. \& Errington, F. P. $(1963 b)$. The size of bacteria, as measured with the Dyson image-splitting eyepiece. J. R. micr. Soc. 82, 39.

Schaechter, M., Williamson, J. P., Hood, J. R. \& Koch, A. L. (1962). Growth, cell and nuclear divisions in some bacteria. J. gen. Microbiol. 29, 421. 